\title{
ADVANCES IN THE TREATMENT OF HEART DISEASE
}

\author{
By Graham W. Hayward, M.D., F.R.C.P. \\ Assistant Physician, St. Bartholomew's Hospital and the National Heart Hospital
}

Apart from the more spectacular recent advances in the surgical treatment of heart disease, both congenital and acquired, medical treatment also has made considerable progress. The additional knowledge of the dynamics of the circulation in heart failure, gained often by the newer methods of investigation, such as cardiac catheterization and the use of radioactive tracers, together with the discovery of new drugs, has led to more effective treatment of heart disease. An attempt is made in this paper to review some of the more important advances.

\section{Congestive Heart Failure}

The degree of absorption from the alimentary tract of the various digitalis preparations varies considerably and only about 20 per cent. of digitalis leaf or tincture is absorbed (Gold, 1946). On theoretical grounds this makes accurate estimation of the dose required difficult and there is a tendency to replace digitalis leaf by one of the purified glucosides. Where rapid digitalization is unnecessary the use of a standardized preparation of digitalis leaf is satisfactory in the majority of patients, but if speed is essential it is preferable to use a purified glucoside, such as digoxin. Absorption from the alimentary tract is more rapid and complete and a dose of 2 to $3 \mathrm{mg}$. by mouth in patients with auricular fibrillation will result in slowing of the heart rate in from 2 to 3 hours. For an even quicker response, digoxin I.O to $\mathbf{I} .5$ mg. may be given intravenously (Evans et al., 1948).

It has long been known that patients with heart disease excrete sodium poorly and the studies of Warren and Stead (1944) have shown that the ingestion of large amounts of salt will cause the reappearance of oedema in patients who have recovered from congestive heart failure. In spite of the present conflict of opinion as to the significance of this salt retention in relation to the dynamics of congestive failure, from a practical point of view there is no doubt that the production of sodium depletion by restricting the dietary intake of salt represents a notable advance in the treatment of the oedema of congestive heart failure. Schemm (1942) advocated a low sodium, acid-ash diet with free administration of fluids and reported good results in patients with oedema. This diet is troublesome to arrange and almost equally good results can be obtained with a diet containing not more than $1.5 \mathrm{gm}$. of sodium chloride. The diuresis resulting from such a diet is aided by the free administration of fluids, up to 3 litres daily being allowed.

Sodium depletion can be ipcreased by giving mercurial diuretics, and this is the basis of their use as diuretics. Reaser and Burch (1946) have shown by means of radio-sodium tracer studies that the mercurial diuretics greatly increase the loss of sodium in the urine and that an oedematous patient may lose as much as $15 \mathrm{gm}$. of sodium in a week. The combined use of a diet low in sodium and the regular use of mercurial diuretics makes the more severe dietary restriction of sodium 0 unnecessary. A word of caution is necessary. If $\varnothing$ there is any evidence of renal impairment, fevero or undue sweating, it is possible to produce symp-co toms of salt depletion, such as muscle cramps, $\stackrel{\rightleftharpoons}{\rightleftharpoons}$ colic, nausea and vomiting with a rising blood urea, and Soloff and Zatuchni (1949) report four deaths which they attributed to this cause. A dose of mersalyl $0.5^{-2}$ cc. once or twice weekly is usually adequate and it is safer and as effective to give it intramuscularly instead of intravenously. Ammonium chloride gr. 30 may be given three times on the day of injection and will increase the diuresis by abouc 20 per cent., although its irritant action on the stomach may preclude its use in severely ill patients.

An increase in body weight of from 8-ro lb., due to the accumulation of extracellular fluid, must occur before clinical signs of oedema or pulmonary congestion appear. Although such a patient will have increasing dyspnoea on exertion, mersalyl is often withheld because of the absence of physical signs of pulmonary congestion; its use at this stage will cause a diuresis with marked subjective improvement. The frequency of injection can be judged by keeping a careful watch on the weight of the patient, increasing weight being an indication for increasing the frequency of injections.

By a combination of the use of digitalis, low salt diet and the regular use of mersalyl the majority of patients with congestive heart failure can be kept 
relatively free from oedema. In long-standing cases, particularly where there is tricuspid insufficiency and gross hepatic congestion, ascites and dependent oedema may be intractable. These patients often suffer from hypoproteinaemia and loss of tissue protein, the result of impaired intake from poor appetite, inadequate formation of plasma protein due to congestion or cirrhosis of the liver and often protein loss from repeated paracentesis. A degree of hypoproteinaemia is thus produced which will contribute to the persistence of oedema. In such a case it is worth trying the effect of a high protein (200-300 gm.) diet with protein supplements, such as casein or Prosol. Correction of this protein deficiency will often result in improvement in the nutrition of the patient, and if the plasma proteins become normal the oedema may respond better to other methods of treatment. Acupuncture may be necessary, the patient being placed in a cardiac bed with feet dependent for I-2 days previously. It is interesting that if mersalyl is given when the flow of fluid from the punctures has : lmost ceased the flow will often recommence.

Acute pulmonary oedema occurs most commonly in cardiological practice as a result of acute left ventricular failure. Recent work on the pathogenesis of this condition has tended to discount the usual mechanical theory of blood accumulating behind the failing left ventricle and has stressed the importance of reflex or neurogenic factors which by causing capillary dilatation and increased permeability are responsible for the outpouring of oedema fluid into the lung (Luisada, 1940). Clinically, there is no doubt that the majority of patients give a history of chronic pulmonary congestion with dyspnoea and orthopnoea and that the methods of treatment previously outlined will diminish or abolish the attacks of acute pulmonary oedema by diminishing the pulmonary congestion. In the treatment of the actual attack the rationale of the various methods of treatment is now better understood. Oxygen is helpful because the pulmonary capillaries become more permeable as a result of anoxia, and pulmonary oedema itself will cause anoxia. Administration of oxygen-at a rate of 6-8 litres by B.L.B mask-will help to break this vicious circle. Morphia, traditionally the drug of choice, acts partly by relieving the restlessness of the patient and partly by decreasing the activity of those pulmonary vasomotor reflexes which increase capillary permeability. A similar result can be obtained using the barbiturates. Nembutal or soneryl at night may abolish the nocturnal attacks, and in case of emergency intravenous injection of one of the rapidly-acting barbiturates may stop an attack. In patients who are having frequent attacks the regular use of phenobarbitone in doses of 4-6 gr. in 24 hours is advised. Probably the most valuable drug in the treatment of an attack is theophylline ethylene-diamine given intravenously (0.24-0.48 gm.). This causes an abrupt fall in right auricular pressure and, in addition, will relieve the bronchospasm, which is an important contributory cause of the acute respiratory distress.

Heart failure caused by chronic lung disease, particularly emphysema, is well known to respond poorly to treatment and when failure is well marked complete recovery is unusual. In these cases, where the cardiac output is above normal, digitalis is theoretically contraindicated as it lowers both the venous filling pressure and the cardiac output (Howarth et al., 1947). In practice, although digitalis rarely produces any dramatic improvement, it may be of value as an adjunct to the usual treatment with oxygen and mersalyl, particularly in those cases where ischaemic or hypertensive heart disease coexists. In such cases slow digitalization over a period of 4-7 days is advisable. Ouabaine (g.strophanthin) may be of value in the treatment of high output failure, as it will increase the output of the heart by direct stimulation of the myocardium. It has the dis-? advantage of being irregularly absorbed from the alimentary tract, so that repeated intravenouso injections are necessary and its pressor effect, if marked, may be undesirable (McMichael, 1948).

\section{Hypertension}

The variable course of benign essential hypertension and the difficulty in giving a prognosis in any individual case makes the assessment of the value of any particular line of treatment, either medical or surgical, difficult. In recent years interest has largely centred on the various surgical procedures which have been devised for the relief of hypertension. Most of these involve removal of the sympathetic chain and ganglia in the lower dorsal and upper lumbar regions. The difficulty in deciding on the worth of the operation has been the absence of information on the course of comparable groups of patients treated medically. Keith, Woolf and Gilchrist (1949) pointed out these difficulties and attempted to overcome them by studying a group of $15 \mathrm{I}$ patients, of whom 96 were treated medically and 55 surgically. Matched groups of patients were constructed as nearly comparable as possible according to their criteria and the results after one year are reported. The most striking difference between the medical and surgical groups was the marked subjective improvement in those patients treated surgically, many of whom were completely free from symptoms. An average fall in diastolic pressure of 
only $8 \mathrm{~mm}$. $\mathrm{Hg}$. was seen in the surgical group, whereas in the medical group an average rise of $8 \mathrm{~mm}$. Hg. occurred during the same period. Cases followed up for longer periods in other clinics have shown in most cases a disappointing fall in blood pressure, and if the purpose of the operation is the cure of hypertension it is at best only moderately successful. One of the chief difficulties is the absence of any reliable test to pick out those cases in which an appreciable fall in blood pressure is likely to be obtained. The commonly used sodium amytal test is unreliable and the use of sympathetic ganglion blocking drugs, such as tetraethyl ammonium bromide, which might be expected to give results comparable to those obtained by surgical sympathectomy, is little better (Hayward, I948).

In malignant hypertension the prognosis with medical treatment is almost uniformly bad and most cases run a short course. Sympathectomy in these cases, although it rarely results in a marked fall in blood pressure, will often lengthen life and alleviate symptoms. In a series of cases reported by Peet and Isberg (1948) 23 patients were alive 5 years after operation. In a successful case vision may be improved and papilloedema disappear, while the marked subjective improvement makes the operation the treatment of choice once the diagnosis has been established.

At the present time it seems fair to state that surgery holds a very limited place in the treatment of hypertension. Those cases most likely to improve are (a) malignant hypertension, (b) patients with severe and intractable hypertensive headache who are likely to get relief of headache by operation even in the absence of a significant fall in blood pressure, and (c) young patients with marked hypertension and left ventricular hypertrophy in whom it is thought the disease is likely to run a short course.

As an alternate to surgical treatment, there has been much interest recently, particularly in the United States, in the dietary treatment of hypertension. Kempner (1946) thought the damaged kidney cells in hypertension could only eliminate with difficulty the breakdown products of animal protein, and that by the use of vegetable proteins only the work of the kidney would be reduced. His diet consists of rice, sugar and fruit only, with vitamin and iron supplements, and provides 2,000 calories daily (460 gm. carbohydrate, $20 \mathrm{gm}$. protein, $5 \mathrm{gm}$. fat). Its sodium content is about $160 \mathrm{mgm}$. The results reported show in many cases relief of symptoms with a fall in blood pressure, decrease in size of the heart and in the extent of the retinal changes. The results have been criticized on the grounds that there was no adequate control group of cases and that many patients failed to adhere to a diet which is admittedly exceedingly monotonous. The value of this degree of protein deprivation in hypertension is not generally accepted and it is probable that many of the good results of the treatment were due largely to the low sodium content of the diet. Bryant and Blecha (1947) obtained equally good results with a diet containing $200 \mathrm{mgm}$. of sodium and $70 \mathrm{gm}$. of protsin daily.

Dietary treatm ent of hypertension is safe and should be tried in those pitients in whom sympathectomy is contemplated. The necessity for protein deprivation is not proved, and a diet containing $70 . \mathrm{gm}$. protein with $200 \mathrm{mgm}$. sodium ( $0.5 \mathrm{gm}$. sodium chloride), aided by the sodium diuresis which mersalyl will often produce in these oedema-free patients, is worth trial. If any improvement occurs in 4-6 weeks, a longer trial should then be carried out.

Many reports have appeared in the literature on the cure of hypertension by nephrectomy in patients with unilateral kidney disease. Langley and Platt (1947) advise pyelography in hypertensive patients under the age of forty-five, particularly in those patients in whom there is no family history of hypertension. If unilateral kidney disease clearly exists and the total renalo function is norm.al, nephrectomy offers a reasonable chance of cure.

\section{Coronary Thrombosis and Myocardial Infarction}

The high incidence of thromboembolic complications in patients who have had a coronary thrombosis was emphasized by Nay and Barnes (1945). These complications occurred in 37 per cent. of their 100 cases, the most common being a second thrombosis or an extension of the infarct (I5 per cent.) and pulmonary embolism (I4 per cent.). In four cases these complications were the cause of death and in eight they were a contributory cause. Many reports, mostly from the United States, had suggested that the use of the anticoagulant drugs heparin and dicoumarol diminished the frequency of thromboembolism and lowered the mortality. The report of the committee set up by the American Heart Association confirms this view (Wright et al., 1948). A series of 800 cases was investigated, divided into comparable 'control' and 'treated' groups. The death rate was 24 per cent. in the control group and 15 per cent. in the treated group, the reduction in mortality being due to the lower incidence of thromboembolic complications. Such complications occurred in 25 per cent. of the control cases, as compared with II per cent. in the treated cases, the incidence of new infarcts, pulmonary, cerebral and peripheral emboli all being lower. These 
figures give strong support to the view that the anticoagulant drugs should be given to all cases of coronary thrombosis where adequate laboratory facilities for the control of the treatment exist. No comparable series' has been published in this country, but it is generally thought that the incidence of thromboembolic complications in patients treated conservatively is considerably lower than that in the 'control' American group. There is undoubtedly a place for anticoagulant treatment in the management of patients with a coronary thrombosis and a more extensive trial in this country is justified.

The time of greatest incidence of thromboembolism is during the second week and most of these complications will occur between the fourth and twentieth day, so that treatment should be started as soon as possible and carried out for at least three weeks. Dicoumarol is potentially a dangerous drug, and it is essential that laboratory facilities should be available for the accurate daily estimation of the prothrombin content of the blood throughout the course of treatment. If such facilities are not available, dicoumarol should not be used.

The aim of treatment is to reduce the prothrombin content to about 20 per cent. of normal and to maintain it at this level for three weeks. Thrombosis on the endocardial surface of the infarct or in the leg veins will occur infrequently under these conditions and the patient will be spared the risk of an embolus. Dicoumarol takes from 2-3 days to lower the prothrombin level. If treatment is started within 24 hours of the infarct, this is no disadvantage, as complications are infrequent before the fourth day and death during the first few days, due to the extensiveness of the infarct cannot be prevented by anticoagulants. If the onset of treatment is delayed beyond the second day heparin should be given as well, to obtain immediate control over the clotting of the blood until the dicoumarol becomes effective. It has the advantage of rapidity of action, but its disadvantages are its high cost and the need for repeated intravenous injections.

The Mayo Clinic scheme is the most satisfactory way of judging the proper dose of the drug. On the first day $300 \mathrm{mgm}$. is given by mouth and prothrombin estimations are done each day subsequently before the dose for that day is decided on. Usually $200 \mathrm{mgm}$. is given on the second day and on subsequent days a dose of 50-200 $\mathrm{mgm}$. whenever the prothrombin level is above 20 per cent. of normal. If the level is below 20 per cent., no dicoumarol is given. In this way it is usually possible to maintain the level at about 20 per cent. throughout the course. Control is not always easy, as the individual response to the drug is variable, and so daily prothrombin estimations are essential. Heparin in a dose of $50 \mathrm{mgm}$. intravenously every four hours may be given in addition during the first two or three days of treatment if immediate control is thought necessary.

The most important complication is haemorrhage, usually from the kidney, and daily examination of the urine for red cells should be made. The incidence of haemorrhagic complications is about 12 per cent., but, fortunately, the incidence of serious haemorrhage is much lower (Wright et al., 1948). The treatment of haemorrhage during dicoumarol treatment is transfusion of fresh blood and the intravenous injection of 64 mgm. of synthetic vitamin $K$. Haemorrhage occurring during heparin treatment is usually controlled by stopping the heparin injections, when the blood coagulation will return to normal within a few hours. More immediate neutralization of its action can be obtained by giving protamine intravenously.

\section{Pulmonary Embolism}

Pulmonary embolism occurs more frequently in medical than in surgical wards, so that the problem of its prevention and treatment is a mattee of concern to the physician. The source of the embolus is nearly always the calf veins where phlebothrombosis or thrombophlebitis has occurred. Elderly patients with congestive heare? failure are particularly likely to develop this complication, probably as the result of stasis and sluggish circulation in the legs, and many patients with so-called hypostatic pneumonia have, in fact, had a small pulmonary embolus. Prophylactic treatment includes regular daily leg exercises and the earlier mobilization of elderly cardiac patients. Few British physicians would subscribe to one current American view that the risk of pulmonary embolism in such patients is so great when they are confined in bed that prophylactic ligation of both femoral veins is necessary. If a pulmonary embolism occurs and the patient survives the initial episode, immediate treatment with heparin and dicoumarol should be instituted on the lines previously described. In this way the 44 per cent. chance the patient has of having a second embolus can be largely avoided (Nay and Barnes, 1945). Similarly, if phlebothrombosis or thrombophlebitis is detected in a cardiac patient the anticoagulants should be given. The daily examination of the legs for a positive Homan's sign-pain in the calf on forced dorsiflexion of the foot-is advised.

\section{Arterial Embolism}

Although surgical removal of an accessible 
embolus will remain the treatment of choice in young patients with a healthy arterial tree, medical treatment, particularly in elderly patients with arteriosclerosis, gives results which are at least as good as those obtained by surgery. The aims of medical treatment are relief of arterial spasm caused by the embolus and the prevention of secondary intra-arterial thrombosis.

Relief of spasm can be obtained by intravenous papaverine, together with paravertebral block in the case of leg emboli or a stellate block if the arms are affected. Thrombosis may be prevented by the immediate administration of heparin and dicoumarol according to the plan already outlined.

\section{Subacute Bacterial Endocarditis}

The introduction of penicillin treatment in this disease has completely altered the prognosis, and what was previously almost always a fatal disease now responds to treatment in the majority of cases. The number of failures on penicillin is still too high and many new problems which have arisen remain to be solved. The reports of the Penicillin Trials Committee of the Medical Research Council - (Christie, 1948, 1949) give valuable information as to the present position of this method of treatment. In the earlier report 49 per cent. of the 269 cases died, but many of them received inadequate treatment. Seven per cent. of these cases receiving the then recommended dose of 500,000 units daily for four weeks relapsed or died infected and in a similar group treated later 20 per cent. died infected or relapsed ; 35 per cent. of those whose infection was controlled died, mostly from heart failure. The most important factor influencing the prognosis was the presence of heart failure at the beginning of treatment. Of those with moderate failure 75 per cent. died, and with severe failure all died. Poor nutrition and a long period of infection before treatment similarly made the prognosis worse. The coefficient of resistance (C.R.) of the infecting organism is important, as the relapse rate rises with increasing resistance.

The present recommended dose is 2 million units daily for 4-6 weeks in those cases with an infecting organism whose C.R. is less than 8; with more resistant organisms a dose of from 10-40 million units may be necessary. In these resistant cases the penicillin level of the blood may be raised by using simultaneously a drug such as Caronamide, which delays the excretion of penicillin by the renal tubules (Boger et al., 1947). The penicillin blood level obtained with 2 million units daily, together with Caronamide (gm. 12-24 daily by mouth), could only otherwise be reached with I0-20 million units daily. The nutrition of the patient should be maintained by a high caloric high protein diet, any anaemia being corrected by iron. Heart failure should be treated from the onset on orthodox lines. Recently many bacteriologically negative cases have been seen in whom repeated blood cultures have failed to demonstrate the infecting organism which, however, has been recovered from the heart valves at autopsy. Such cases respond poorly to penicillin and the higher dosage scheme should be adopted.

In the prevention of bacterial endocarditis it is important to prevent a bacteraemia occurring in patients with damaged heart valves. This most commonly occurs after dental extraction and it is wise to have this carried out under penicillin cover in patients with rheumatic or congenital heart disease. The injection of procaine penicillin, I cc. (300,000 units) on the day of extraction and for two days subsequently, probably provides adequate protection.

\section{Syphilitic Aortitis}

The efficiency of penicillin treatment of many syphilitic lesions has naturally led to its use in the treatment of syphilitic aortitis and aneurysm. It is too soon to assess the ultimate value of this line of treatment, but if used with care the immediate results are satisfactory and untoward reactions are rare. The same precautions apply to penicillin as to any potent anti-spirochaetal drug and the risks of sudden weakening of the wall of the aortaor narrowing of the coronary ostia have to be considered. It must also be remembered that the fibrosis which accompanies healing may increase the degree of aortic regurgitation or further narrow the mouths of the coronary vessels. The most favourable cases are those under the age of fifty, with no history of cardiac pain or heart failure and preferably without aortic regurgitation. A period of 2-3 months' preparation with bismuth and iodides should precede penicillin treatment and the patient should be admitted to hospital for the course of injections. A total dose of 8-12 million units given over the course of ro days is adequate. Herxheimer reactions are uncommon (Moore, 1947), but careful watch should be kept for the onset of congestive failure, chest pain or evidence of E.C.G. or radiological deterioration. Any of these should lead to the immediate cessation of treatment.

\section{Thyroid Heart Disease}

The use of thiouracil derivatives enables the symptoms of hyperthyroidism to be controlled in nearly all cases and the basal metabolic rate is restored to normal. The importance of this in the treatment of thyroid heart disease is obvious, whatever may be one's view on the relative merits of medical and surgical treatment. Although the symptoms and signs of thyroid heart disease may 
be improved by these drugs, and patients with auricular fibrillation revert to normal rhythm, the indefinite length and uncertainty of medical treatment are serious disadvantages in cardiac cases. The chief value of these drugs is in the preoperative preparation of patients so that an interim thyroidectomy may be safely carried out in a patient who shows no signs of hyperthyroidism. Methyl thiouracil is given, $300 \mathrm{mgm}$. daily at first, later reduced to a maintenance dose of 50-100 mgm. daily. The risk of agranulocytosis with these relatively low doses is small and treatment is controlled by periodic estimations of the basal metabolic rate. The drug is stopped for two weeks before operation, Lugol's iodine, minims I 5-30, being given daily during this period.

The thiouracil drugs, if given in larger doses to patients without hyperthyroidism, will produce hypothyroidism. This makes them occasionally of value in cardiological practice in patients in whom a low metabolic rate may be desirable. Good results have sometimes been seen in severe angina, auricular fibrillation which is difficult to control with digitalis, chronic cor pulmonale and more rarely in intractable heart failure. The higher doses sometimes necessary-500-800 mgm. dailymakes the risk of agranulocytosis greater and frequent estimations of the white cell count are necessary.

\section{Myxoedema Heart Disease}

Myxoedema heart disease is one of the rare types of reversible heart disease and administration of thyroid gland will cause disappearance of all cardiac symptoms and signs. It is not generally realized that these patients are initially hypersensitive to thyroid. Too vigorous treatment in the early stages frequently causes anginal pain in older patients and leads to the abandonment of treatment. The initial dose should be gr. $\frac{1}{4}-\frac{1}{2}$ of dried thyroid daily for 2-3 weeks, later increasing the dose, but keeping the basal metabolic rate at about - Io to - I 5 per cent.

Further advances in the treatment of heart disease may be expected in the near future, particularly in the field of cardiac surgery for conditions other than congenital malformations of the heart. This is illustrated by the recent reports of successful resection of the left auricular appendage in patients with recurrent arterial emboli (Madden, 1949) and anastomosis of the azygos and pulmonary veins for relief of pulmonary congestion in patients with 'tight' mitral stenosis (Bland and Sweet, 1949).

\section{BIBLIOGRAPHY}

BLAND, E. F., and SWEET, R. H. (1949), 7. Amer. Med. Ass. 140, 1259 .

BOGER, W. P., KAY, C. F., EISMAN, S. H., and YEOMAN, E. E. (1947), Amer. . Med. Sc., 214, 493.

BRYANT, J. M., and BLECHA, E. (1947), Proc. Soc. Exper. Biol. $N . Y ., 65,227$.

CHRISTIE, R. V. (1948), Brit. Med. F., r, I.

CHRISTIE, R. V. (1949), Ibid. In press.

EVANS, W., DICK, P., and EVANS, B. (1948), Brit. Heart $\mathcal{f}$. I0, 103.

GOLD, H. (1946), F. Amer. Med. Ass., 132, 547.

HAYWARD, G. W. (1948), Lancet, I, 18.

HOWARTH, S., McMICHAEL, J., and SHARPEY-SCHAFER, E. P. (1947), Clin. Sci., 6, 187 .

KEITH, M. A., WOOLF, B., and GILCHRIST, A. R. (1949) Brit. Heart $¥$., $11,287$.

KEMPNER, W. (1946), Bull. N.Y. Acad. Med., 22, 358.

LANGLEY, G. J., and PLATT, R. (1947), Quart. F. Med., 16, 14

LUISADA, A. (1940), Medicine, 19, 475.

MADDEN, J. L. (1949), Э. Amer. Med. Ass., 140, 769.

McMICHAEL, J. (1948), Brit. Med. F., 2, 927.

MOORE, J. E. (1947), Proc. Roy. Soc. Med., 40, 811 .

NAY, R. M., and BARNES, A. R. (1945), Amer. Heart f., 30, 65

PEET, M. M., and ISBERG, E. M. (1948), Ann. Int. Med., 28, 755.

REASER, P. B., and BURCH, G. (1946), Proc. Soc. Exper. Biol, N.Y., 63, 543.

SCHEMM, F. R. (1942), Ann. Int. Med., 17, 952.

SOLOFF, L. A., and ZATUCHINI, J. (1949), भ. Amer. Med. Ass., 139, 1136 .

WARREN, J. V., and STEAD, E. A. (1944), Arch. Int. Med., 73 138 .

WRIGHT, I. S., MARPLE, C. D., and BECK, D. F. (1948), Amer. Heart F., 36, 801 . 